\title{
Peter Emerson (ed): Designing an all-inclusive democracy
}

\author{
Springer-Verlag, Berlin, 2007
}

\author{
Donald G. Saari
}

Received: 12 October 2009 / Accepted: 12 October 2009 / Published online: 27 October 2009

(C) The Author(s) 2009. This article is published with open access at Springerlink.com

Somewhere in his writing, Condorcet stressed a reality that we often ignore: finding suitable voting rules is only a part of what needs to be done to ensure "good election decisions." Over a couple of centuries ago, Condorcet tried to educate us about the need to combine the search for an appropriate decision rule with that of having an educated and informed society. After all, a voting rule is merely a tool; if even the best possible tool is being applied by uninformed users to nonsense, expect nonsensical outcomes. Therefore, equally important as the identification of pertinent voting rules is the need to create ways that will generate informed users (voters) and carefully considered options.

This may sound idealistic to those of us who are veterans of far too many departmental meetings - where we may have colleagues who doggedly hang on to extreme views. With all of our scars, we may question whether it is possible to achieve the desired state of an informed populace, where people actually listen to others. Oh, we know what needs to be done; we need to encourage considered discussions and deliberations; we need to design an appropriate dynamic to encourage voters to compromise and reformulate proposals to attain acceptable outcomes.

The approach can work, probably all of us can provide examples illustrating how, after thoughtful discussions, modified versions of initial proposals emerged that better addressed the concerns of all. On the other hand, we also can point to more destructive examples where, rather than a contemplative review, the result of deliberations just solidified divisive stands. What makes the difference? Could the choice of a voting rule play a role? Could it be that some voting methods encourage intransigence and provide opportunities for division, while others provide appropriate incentives/

D. G. Saari $(\bowtie)$

Institute for Mathematical Behavioral Science, University of California,

Irvine, CA 92697-5100, USA

e-mail: dsaari@uci.edu 
disincentives to encourage cooperation? These questions capture the major theme of the book under review.

This issue of a dynamic to encourage voters to reconsider options may be ignored by some readers of this review if only because it appears to be outside of the scope of our interests/expertise. I disagree; more is said about this below. However, the one person who has made the combination of these two themes (which could be called the "Condorcet program") his life's work is the editor and main author of the book under review: Peter Emerson. As Emerson has actually done, it is easy to imagine him stepping off a plane in a region troubled by conflict, unfolding his ever-present bike, and peddling off to some hot-spot to meet with opposing sides to try to promote reason. He lives to advance his cause of encouraging civility and reason by reforming "voting." He proposes doing so with procedures to encourage reconsideration and interactions that are supported by using an appropriate voting rule. Important to this review is that the proposals Emerson puts forth in the first part of the book are based on, and have been tested by, pragmatic experience.

Emerson combines techniques for the dynamics of a group interaction with a voting rule; the intent of this mixture is to engender a civil discourse leading to more useful group decision outcomes. More important than his particular choice of a dynamic for group interactions (which is fairly standard) is that there is a dynamic. It is the dynamic that allows for interactions and compromise, it is the dynamic that permits review and reconsideration of proposals. Rather than his outlined choice, any group dynamic that encourages reasoned thought and the introduction of revised or new proposals while discouraging intransigent stands should suffice. The key ingredient in this mix is the voting rule.

Above, when discussing our common experiences of cooperative and divisive discussions in meetings, I wondered whether the choice of a voting rule can make a difference. Emerson believes so. Throughout this book, you will discover concrete examples (often found in his footnotes; do not miss them!) of where society has screwed up probably because of the choice of a voting rule. Emerson uses these opportunities, supported by several created examples, to discuss what could have been done instead. His basic theme is captured by the "Democracy is for everybody, not just a majority" comment. In promoting this thought, he argues how the pairwise vote and the plurality vote introduce an "either-or" divisive sentiment that can damage not only a consideration of the minority input, but also discourage a group from trying to reach an outcome that would be of more universal appeal.

After some thought, his point becomes obvious. He is arguing that with such rules, a majority can always have its way, so it need not even bother considering the views of a minority. While this concern reflects the long standing fear about the "tyranny of the majority;" what Emerson adds to the discussion is how the choice of a voting rule can become a willing accomplice to this threat; whenever a rule provides the majority the ability to ignore the views of a minority, it could even encourage tyrannical behavior. Emerson's claim is that many of our voting systems, such as the pairwise and plurality vote, do precisely this, and he supports his arguments with examples.

However, is this an unfortunate reality of life, or can any positive news be found? Does there exist a voting system that punishes extremes and encourages a consideration of the views of others? Emerson thinks so; he claims that the Borda Count (where 
an $n$-candidate ballot is tallied by assigning $n-j$ points to a $j$ th ranked candidate) is a crucial component of the solution. From his personal, pragmatic experiences, he asserts that the Borda Count encourages compromise; when the Borda Count is used in a dynamic of proposals and counterproposals, he believes that more acceptable group outcomes emerge. Although his arguments are supported by several examples, the associated theoretical concern, of course, is to find support or evidence to the contrary. For instance, why the Borda Count? Could not other rules work just as well? All of this is addressed later in this review.

Of course, there are several practical issues, such as where a voter cannot, or will not, rank all of the alternatives. Here, Emerson proposes different approaches; an obvious one, which has been proposed by many others, is that if a ballot ranks only $k<n$ of the candidates, it should be tallied by assigning $k+1-j$ points to the $j$ th ranked candidate and zero for all candidates who have been left off of the ballot. (So, a truncated ballot listing only $A \succ B \succ C$ out of six candidates would be tallied by assigning 3,2,1 points, respectively, to $A, B, C$, and zero to all others.) In fact, this approach captures Borda's own description of his rule where the number of points assigned to a candidate equals the number of lower ranked candidates on the ballot. (So, in tallying a truncated ballot, all non-ranked candidates are lumped at the bottom.)

Through this book, Emerson is speaking to several audiences among them are those of us in social choice who are interested in theory. (Reading the book, it is easy to find all sorts of new issues to consider.) Perhaps the audiences that he wants most to influence include those who are exploring reform and those who might want to use these methods. As such, much of his description is devoted toward practical "nutsand-bolts" descriptions, such as how to more easily tally ballots (and an instructive $\mathrm{CD}$ comes with the book). This explanation is necessary to include for these particular audiences, but I must confess that I found it distracting enough to delay my completing the reading of the book.

It is natural to wonder whether Emerson's claims of the effectiveness of the Borda Count and the variants will stand the scrutiny of a theoretical investigation. This leads to a particularly valuable part of the book that must not be missed: it is the one-two punch of successive chapters written by Maurice Salles and by Hannu Nurmi. In a short number of pages devoted to each of their chapters, they nicely capture the intellectual history, theoretical developments, and controversies of a sizeable portion of the debate between Borda's and Condorcet's approach.

Salles describes the initial victory of Condorcet's approach over Borda's; a victory that was reconfirmed by the work in the 1940s and 1950s by Black and Arrow. What I liked about this review is how Salles identifies certain intellectual aspects of the development of the support for majority voting starting from the work of Condorcet and continuing up to Arrow's seminal result. (Of course, at this point I must remind the reader that we now know why Arrow's result holds (Saari 2008); one of his assumptions totally emasculates another crucial one. However, if a crucial assumption that we thought was being used is not, it means that Arrow's conclusion does not mean what we have normally thought it did.) There is a much to be found in these pages including descriptions of the rebirth of interest in the Borda Count, providing examples (including the 2002 French presidential election) to illustrate points, and Salles 
also addresses why economists found the Arrow-type of assumptions to be of interest. A lot of information is packed into a few pages!

Nurmi picks up from where Salles ends to address strengths and weaknesses of the Borda Count. In his inimitable manner, Nurmi creates clever examples to identify weaknesses in Emerson's proposed methods that differ from the pure Borda Count. This, of course, raises the unexplored research question about the likelihood of these faults. (They can be serious if all voters opt for the alternative options.) In order to come to grips with Emerson's theme of comparing the dangers of the pairwise vote with the advantages of the Borda Count, Nurmi weaves in results developed by many researchers in the area, such as Fishburn's estimates about how likely it is to have a Condorcet cycle, and so forth. Central to his analysis is the long-standing criticism that the Borda Count need not elect the Condorcet winner. While this comment is standard, it is worth wondering whether a more accurate criticism is that the Condorcet approach need not elect the Borda winner. Indeed, by appealing to recent theoretical results that identify the source and explain all possible differences between the outcomes of these two approaches, which Nurmi illustrates with nice examples that have become a trademark of his writings, he cautiously suggests why we should not be enamoured with the Condorcet winner.

There remains a gap in this critique and an analysis of Emerson's proposals; the reader might be wondering how can the choice of a rule guide the dynamic group process toward more acceptable societal outcomes? This issue is addressed through the book in an intuitive manner supported by the analysis of several examples, but it is not dealt with in a theoretical way. As such, it is worth suggesting how to reformulate these concerns into a framework that can be examined theoretically. Assistance comes from the concept of a core in spatial voting where McKelvey's seminal "chaos theorem" (1987) proves that whenever the voters' ideal points define an empty core, this situation can create a wildly divisive effect on deliberations. Recall, an empty core means that there are several different winning coalitions, each of which can propose significantly different choices leading to the possibility that almost anything can happen! Indeed, the outcome could be even something nobody really wants. With an empty core, then, it is worth anticipating a lack of collegiality with the possibility of disappointing societal outcomes. On the other hand, should a core exist, it loosely plays the role of an attractor on the group dynamics of interactions; namely, to ensure that a new proposal can beat a previous one, the new choice should be in, or at least closer to, the core. A way to rephrase and examine Emerson's assertions, then, is to determine which (if any) positional methods admit a core.

It turns out that these questions have been explored. (While they were not motivated by Emerson's concerns, they should have been because, after the fact, the parallels are obvious.) In a recent paper (Saari 2010), it was shown, for instance, that the plurality vote, or the antiplurality vote, or many other voting rules, always have an empty core; thus with these rules, one should not be surprised about somewhat divisive behavior where different winning coalitions can move the outcome away from what would be considered societally more acceptable. In fact, the theoretical dynamics of what can occur is not dissimilar to Emerson's descriptions of could happen. On the positive side, the only rule that avoids this problem by allowing a core is the Borda Count. This means, as Emerson discovered from experience, that if the dynamic is guided by 
determining outcomes with the Borda Count, then, in order for new sets of proposals to have a chance against the previous ones, they have to converge toward the core. Again, this corresponds to what Emerson claims he has observed.

There are many other interesting aspects of this book. You definitely should read the "Foreword" by Michael Dummett, where he nicely identifies what he views as the guiding principles of Emerson's efforts. In a short chapter, Christine Bell critiques Emerson's work more from the perspective of those important concerns that might be found on editorial pages; e.g., in terms of questions about self-determination and human rights. In the chapter written by Phil Kearney and Aileen Tierney, the earlier theme of generating new proposals, the role of consensus, and inclusive decisionmaking and the negative effects of pairwise comparisons are reexamined in terms of the literature with several examples that I found quite interesting. The varied contents of the book, plus a sharpening of the main message of "finding consensus" is captured by the conclusion written by Emerson with assistance from Elizabeth Meehan.

So, what is my view? There is much to learn from this book, so I suggest that it should be in your bookshelf. Beyond those of us interested in theory, this is a book that should be seriously considered by practitioners involved in group decisions.

Open Access This article is distributed under the terms of the Creative Commons Attribution Noncommercial License which permits any noncommercial use, distribution, and reproduction in any medium, provided the original author(s) and source are credited.

\section{References}

McKelvey R (1987) General conditions for global intransitivities in formal voting models. Econometrica 55:923-934

Saari DG (2008) Disposing dictators; demystifying voting paradoxes. Cambridge University Press, New York

Saari DG (2010) The core with positional spatial voting. Homo Oeconomicus 26(3/4). In: Holler M, Widgren M (eds) Essays in honor of Hannu Nurmi, HOEC (Munich) 